\title{
SAE - Sistema de Apoio Educacional
}

\section{Lorrany Oliveira Souza, Giovanni Almeida Santos, Vandor Roberto Vilardi Rissoli}

Núcleo de Apoio Estratégico e Educacional (NAEEd) - Faculdade do Gama (FGA) Universidade de Brasília (UnB), Gama, DF

Apoio: Fundação de Apoio à Pesquisa do DF e Centro de Educação a Distância da UnB

lolo.souza80@yahoo.com, giovannix@gmail.com, vandorissoli@gmail.br

\begin{abstract}
This paper presents an Intelligent Tutoring System that has been used in different levels and learning modalities, achieving promising results in more personalized education in the Federal District.

Resumo. Este trabalho apresenta um Sistema Tutor Inteligente que vem sendo utilizado em diferentes níveis e modalidades de ensino, alcançando resultados promissores na educação mais personalizada no Distrito Federal.
\end{abstract}

\section{Introdução}

As novas características da época atual demandam de agilidade na compreensão de mudanças que promovem adaptações e/ou evoluções, inclusive em processos tidos como mais tradicionais, como na educação. Essa realidade atinge também as novas possibilidades educacionais de apoio contínuo aos indivíduos interessados em aprender.

Novas tecnologias têm surgido para suprir demandas anteriormente existentes, assim como as novas necessidades pertinentes ao momento da sociedade mais moderna, sendo destacado neste trabalho as tecnologias mais integradas à Educação, principalmente aquela conhecida como Sistema Tutor Inteligente (STI).

Essa tecnologia existe a décadas e consiste em um tipo de software educacional que se adapta às características de seus usuários, mais tradicionalmente, estudantes buscando apreender (reter), em suas estruturas cognitivas, conteúdos novos que fazem parte de um domínio disciplinar específico relacionado a formação desejada.

Os STIs mais modernos têm-se destacado no meio educacional, pois continuam em evolução, procurando atender cada vez mais a diversidade de metodologias educacionais existentes, oferecendo aporte adequado aos seus diferentes tipos de usuário. Dessa forma, este tipo de software educacional se transforma em uma tecnologia mais interativa, que apoia o processo educacional e seus principais participantes, não mais correspondendo a ser um software tutor somente do estudante.

O STI auxilia no sucesso do ensino-aprendizagem, empregando mecanismos e lógicas provenientes da Inteligência Artificial. Esse tipo de software possui recursos que se adaptam às necessidades de seus estudantes, sendo esta adaptação possível pela combinação coerente e dinâmica de informações relacionadas ao estudante, ao domínio ou conteúdo de estudo e ao congruente processo pedagógico que fornecerá apoio significativo aos seus participantes (Psotka, Massey e Mutter, 1988). 
O principal objetivo dos STIs é proporcionar um ensino-aprendizagem sintonizado com as características de seus diferentes alunos, personalizando esse processo com as particularidades cognitivas de cada aprendiz. Nos modernos STIs outros perfis tornamse usuários desse software, como os docentes, que colaboram com as interações mais coerentes e diretas do STI com os estudantes, mas que também obtém um acompanhamento detalhado do comportamento de cada aprendiz, além das inferências realizadas.

Este trabalho está organizado em mais três seções e apresenta algumas características do STI conhecido como SAE na seção 2. Na seção 3 são indicados alguns dos resultados obtidos por este STI, enquanto que na seção 4 são apresentadas algumas conclusões. As principais referências encerram este trabalho.

\section{Métodos}

O Sistema de Apoio Educacional (SAE) foi desenvolvido envolvendo pesquisadores de três universidades brasileiras nos primeiros anos desse milênio, chegando a publicar trabalhos científicos a partir de 2006 sobre esse projeto (Rissoli e Giraffa, 2006).

O SAE é um STI que partiu da arquitetura modular mais tradicional desse tipo de software (Domínio, Aprendiz, Tutor e Interface) e prosseguiu integrando novos módulos em sua estrutura, a fim de atender demandas pertinentes às posturas educacionais que são mais ativas e centradas na aprendizagem discente (Rissoli, 2007).

A Figura 1 procura ilustrar os recursos atualmente implementados no SAE por meio de diferentes módulos que são acionados pelo mecanismo de controle, desenvolvido neste software para fornecer informações pertinentes às diferentes necessidades dos perfis de usuários que o SAE atende (aluno, professor, monitor, diretor de curso, coordenador pedagógico ou secretário do diretor e responsável pelo aluno).

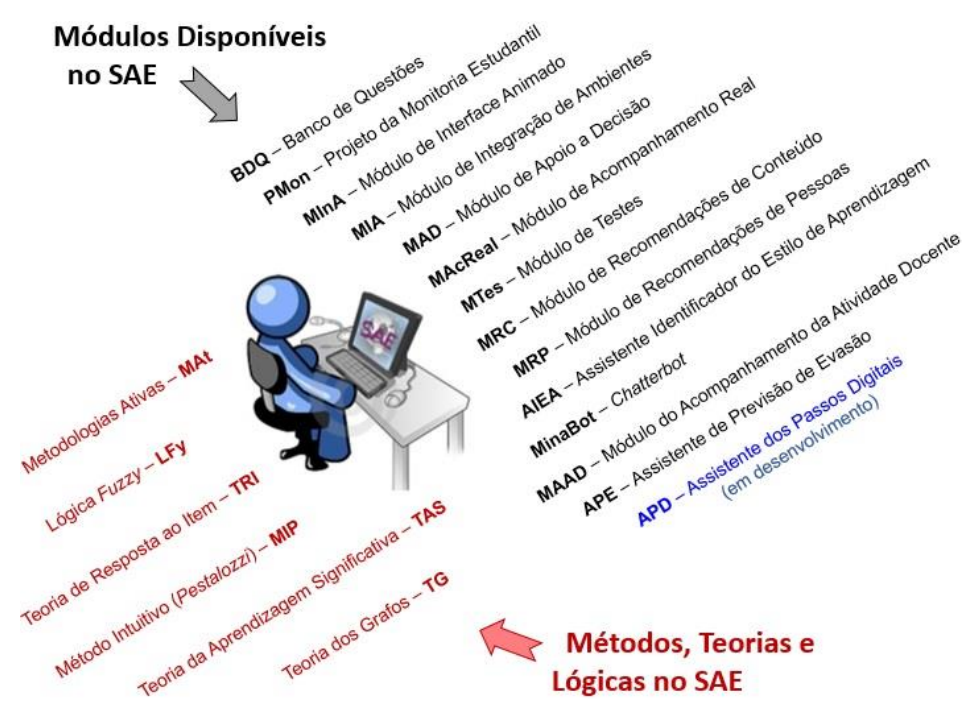

Figura 1. llustração dos métodos e tecnologias atualmente implementadas no SAE.

Diante dessa diversidade de perfis de usuário, o SAE possui um módulo de interface bem variado, pois cada um de seus tipos de usuário recebem assistências mais adequadas à colaboração ou ao acompanhamento do processo educacional, a fim de satisfazer as necessidades de cada um que participa, direta ou indiretamente, da realização desse processo. A assistência do SAE aos seus diferentes perfis acontece em tempo real e o classifica como um Intelligent Teaching Assistant (ITA). 
Segundo Yacef (2002), um ITA é um STI que possui mais recursos de assistência à aprendizagem dos estudantes, além da participação dos docentes como seus novos usuários. Estes novos usuários colaboram com o direcionamento da atuação do STI e na consolidação da "inteligência" do software (aprendizagem de máquina), contribuindo, ainda, com a superação de uma das principais dificuldades desse tipo de software educacional que consiste na coleta de dados difíceis de serem obtidos pelas interfaces tecnológicas disponíveis atualmente. Em contrapartida, o ITA compartilha informações da modelagem dos alunos com seus outros usuários participantes do processo, a fim de que todos possam colaborar com o sucesso e a qualidade desse processo educacional.

O volume de siglas disponíveis na ilustração da Figura 1 procura somente indicar as diferentes implementações existentes no SAE como módulos integrados a arquitetura do STI/ITA. Todos procurando fornecer assistência significativa aos usuários do SAE. Mais de uma dezena de trabalhos de conclusão de cursos de graduação, algumas dezenas de trabalhos de iniciação científica, tese de doutorado e dissertação de mestrado estão envolvidos na evolução contínua desse software educacional que está disponível no Portal do Software Público Brasileiro na comunidade SAE.

O módulo que fornece orientação pedagógica no SAE (MInA) acessa sua base de conhecimento que permite a realização de inferências fuzzy sobre o comportamento de cada estudante usuário do SAE através do acesso ao módulo de domínio (MRC), ao seu Banco de Questões (BDQ) e a Monitoria (PMon), além do Módulo de Integração de Ambientes (MIA) que envolve, atualmente, a interoperabilidade com um Ambiente Virtual de Aprendizagem (AVA - Moodle), caso a disciplina o esteja também utilizando.

$\mathrm{O}$ processo de inferência fuzzy do SAE pode integrar até quatro variáveis linguísticas atualmente (esforço, desempenho, participação e acompanhamento real) para apurar as ações (comportamentos) e os resultados que cada estudante está obtendo sobre os conceitos ou tópicos que compõe o conteúdo de estudo previamente organizado em um Mapa Conceitual (Novak, 1998). Geralmente, os professores da disciplina, acompanhados por uma equipe multidisciplinar, elaboram este mapa que permitirá ao SAE "aprender" sobre o domínio e subsidiar todo processo educacional, fornecendo assistência às ações dos alunos, monitores e docentes, principalmente (agentes diretos).

As orientações pedagógicas fornecidas diretamente aos estudantes pelo SAE estarão baseadas nessas inferências que empregam uma lógica multivalorada (Lógica Fuzzy) capaz de tratar as imperfeições e incertezas inerentes as informações, inclusive, quando manipuladas somente pelos seres humanos (Zadeh, 1979).

\section{Resultados}

O uso do SAE como recurso tecnológico interativo de apoio educacional tem acontecido em diferentes áreas de conhecimento, trabalhando com diferentes níveis educacionais a partir dos anos finais do ensino fundamental até os cursos de pós-graduação, além de diferentes modalidades de ensino (presencial, semipresencial e a distância).

Com o objetivo de sintetizar alguns desses resultados estão sendo citadas as publicações mais recentes que foram realizadas na área da Saúde (Rissoli e Furtado, 2019) e da Engenharia (Nunes, Santos e Rissoli, 2018) que utilizaram o SAE. A satisfação dos participantes (alunos e professores) se destaca nas duas áreas por meio de 
VIII Congresso Brasileiro de Informática na Educação (CBIE 2019)

Anais dos Workshops do VIII Congresso Brasileiro de Informática na Educação (WCBIE 2019)

uma pesquisa aplicada aos estudantes após o final do período letivo nas disciplinas de Bioquímica e Algoritmos no nível superior, respectivamente.

O nível de satisfação das turmas nas amostragens simples propiciadas por esta pesquisa (em formato survey), que convidou a todos para participarem, ficou superior a $90 \%$, com nível de confiança de $95 \%$ nos resultados apurados.

\section{Conclusões}

As reflexões educacionais sintonizadas as características da sociedade atual precisam ser mais interativas, no qual o processo de avaliação demanda de melhor acompanhamento para ser mais condizente com as habilidades e expectativas dos aprendizes. Simplesmente avaliar, de maneira mais tradicional, ao fim de algum ciclo de estudo, não basta, sendo necessário o acompanhamento contínuo para se estabelecer um processo de avaliação que promova o estimulo através da interação e que respeite o ritmo de cada estudante, lhe fornecendo a percepção mais segura de um colega de jornada atento aos diferentes caminhos de aprendizagem que poderão ser percorridos com qualidade.

Dessa forma, a combinação de tecnologias coerentes, com as metodologias educacionais mais adequadas, é fundamental à aprendizagem significativa discente, em que o SAE aparece como alternativa eficiente à realização de um processo educacional personalizado, sem sobrecarregar os docentes, e trabalhando a habilidade social e intelectual com mais autonomia de cada aprendiz.

\section{Referências}

Novak, J. D. (1998) Learning, creating, and using knowledge: Concept maps as facilitative tools in schools and corporations. New Jersey: Lawrence Erlbaum.

Nunes, C. F. D.; Santos, G. A. e Rissoli, V. R. V. (2018) Tecnologia 'Inteligente' no apoio ao Processo Educacional em Engenharia. In: International Symposium on Project Approaches in Engineering Education, Brasília. Proceedings. p. 839-847.

Psotka, J.; Massey, L. D. e Mutter, S. (1988) Intelligent tutoring systems: Lessons learned. Hillsdale, NJ: Erlbaum.

Rissoli e Furtado (2019) Tecnologia 'inteligente' associada à aprendizagem significativa em Bioquímica. In: Leite, C. R. M. et al. (Org.) Novas tecnologias aplicadas à saúde. Mossoró (RN): EDUERN. p.329-350.

Rissoli, V. R. V. (2007) Uma proposta metodológica de acompanhamento personalizado para aprendizagem significativa apoiada por um assistente virtual de ensino inteligente. Tese (Doutorado) - UFRGS. PGIE, Porto Alegre, RS.

Rissoli, V. R. V. e Giraffa, L. M. M. (2006) Aprendizagem Significativa Apoiada por um Sistema Tutor Inteligente usando Lógica Fuzzy. In: SBIE - DF.

Yacef, K. (2002) Intelligent Teaching Assistant Systems. In: International Conference on Computers in Education. Proceedings. New Zeland: IEEE. v.1, p.136-140.

Zadeh, L. A. (1979) A Theory of Approximate Reasoning. In Hayes, J., Michie, D. \& Mikulich, L. (Eds.). Machine Intelligence. Sussex: Ellis Horwood, 9(1), 149-194. 